\title{
Mineração
}

\section{Estudo de variabilidade e otimização do circuito de moagem SAG da Usina do Sossego}

\section{(Optimization of the Sossego SAG mill grinding circuit)}

\author{
Maurício Bergerman \\ Engenheiro de Processo, Vale, Departamento do Cobre \\ E-mail:mauricio.bergerman@vale.com \\ Homero Delboni \\ Professor, Dr., Universidade de São Paulo, Departamento de Engenharia de Minas e de Petróleo \\ E-mail:hdelboni@usp.br \\ Marco Nankran \\ Gerente de Processo, Vale, Departamento do Cobre \\ E-mail:marco.nankran@vale.com
}

\section{Resumo}

A Mina do Sossego, localizada na região sudeste do Estado do Pará, foi a primeira usina da Vale a operar um moinho SAG para processar minério de cobre e ouro. Nos primeiros três anos de operação, foram estudadas alternativas para aumento de produção, principalmente focadas em aspectos operacionais da moagem SAG.

Em seguida, foram realizados estudos visando a identificar o desempenho do circuito em função dos diferentes tipos de minérios alimentados. Uma caracterização completa do minério foi realizada em conjunto com a calibração de modelos matemáticos baseados em amostragens conduzidas no circuito industrial. $\mathrm{O}$ simulador foi então usado para prever níveis de produção associados a cada tipo de minério, assim como determinar configurações otimizadas para o circuito.

Esse artigo descreve em detalhes os principais aspectos da otimização do circuito, assim como o método para prever a produção em função das características do minério e do circuito.

Palavras-chave: Moagem, simulação, caracterização, SAG.

\begin{abstract}
Sossego was the first Vale SAG mill operation to process copper-gold ore. It is located in the State of Para, southeastern Amazon region of Brazil. In the first three years of continuous operation, Vale investigated different alternatives for improving the circuit's performance by investigating operating conditions, mainly focusing on the SAG mill.
\end{abstract}

It was decided to further assess the performance of the comminution circuit as a function of ore characteristics. A comprehensive ore characterization program was then conducted, together with the calibration of mathematical models on the basis of surveys carried out at the industrial circuit. The simulator was then used to predict the throughput associated to each ore type, as well as to establish the optimized circuit configuration and tailored operating conditions.

This paper describes in detail the main aspects of optimizing the industrial circuit performance, as well as the successful method for predicting the production as a function of ore characteristics and circuit configuration.

Keywords: Grinding, simulation, characterization, $S A G$. 


\section{Introdução}

A Mina do Sossego foi a primeira operação de cobre da Vale a entrar em operação. O depósito foi descoberto em 1997 e compreende os corpos denominados Sossego e Sequeirinho.

A mina está localizada a aproximadamente $70 \mathrm{~km}$ de Carajás, próximo à cidade de Canaã dos Carajás. A usina tem capacidade para processar $41.000 \mathrm{t}$ de minério por dia, o que equivale a $15 \mathrm{mi}$ lhões de toneladas por ano, lavradas de cavas a céu aberto. A reserva provada é da ordem de $255 \mathrm{Mt}$ com um teor médio de $1,0 \%$ de cobre e $0,3 \mathrm{~g} / \mathrm{t}$ de ouro.

O minério é um granito com presença significativa de magnetita. Valores típicos de WI variam de 17 a $20 \mathrm{kWh} / \mathrm{t}$, além de possuírem um índice de abrasão relativamente alto, de 0,5 .

A operação da usina iniciou-se em abril de 2004, com uma capacidade instalada de produção de $540.000 \mathrm{t}$ anuais de concentrado, com $30 \%$ de cobre e $8 \mathrm{~g} / \mathrm{t}$ de ouro.

O circuito completo da usina e a estratégia de controle adotada estão descritos em detalhes em Nankran, Bergerman e Miranda (2007). O presente trabalho tem por foco o circuito de moagem.

O produto da mina é alimentado em um britador giratório 6089, com abertura nominal de $140 \mathrm{~mm}$, gerando um produto com $\mathrm{P}_{80}$ de $125-150 \mathrm{~mm}$. O circuito de moagem consiste de uma linha SABC, com capacidade nominal de $1.841 \mathrm{t} / \mathrm{h}$,

O produto da britagem primária alimenta um SAG de $38 \times 23 \mathrm{ft}$, dotado de um motor com potência de 20 MW. A descarga do moinho SAG alimenta duas peneiras vibratórias de 12' x 24', cuja fração retida é encaminhada a uma britagem de seixos (pebbles), composta de dois britadores cônicos, que reduzem o material a um $\mathrm{P}_{80}$ de $12 \mathrm{~mm}$. O produto dos rebritadores retorna ao moinho $\mathrm{SAG}$, fechando o circuito. A fração passante na peneira é bombeada para duas baterias de ciclones de 33', cujo underflow reunido é encaminhado a dois moinhos de bolas de 22' x 32', equipados com motores de $8,5 \mathrm{MW}$ cada. $\mathrm{O}$ produto da moagem, que alimenta o estágio de flotação, apresenta um $\mathrm{P}_{80}$ nominal de 0,21 $\mathrm{mm}$ com $40 \%$ de sólidos. usina.

A Figura 1 ilustra o fluxograma da

Estudos iniciais (Delboni, 2004; Delboni et al., 2006) indicaram potencial de aumento de capacidade, mediante mudanças na granulometria de alimentação, velocidade do SAG, desenho das grelhas de descarga e carga de bolas do moinho SAG.

Posteriormente, em 2006, foi realizada uma alteração no circuito para possibilitar o encaminhamento do produto dos rebritadores diretamente aos moinhos de bolas (Nankran, Bergerman, Fonseca, 2007). Essa medida visou a um

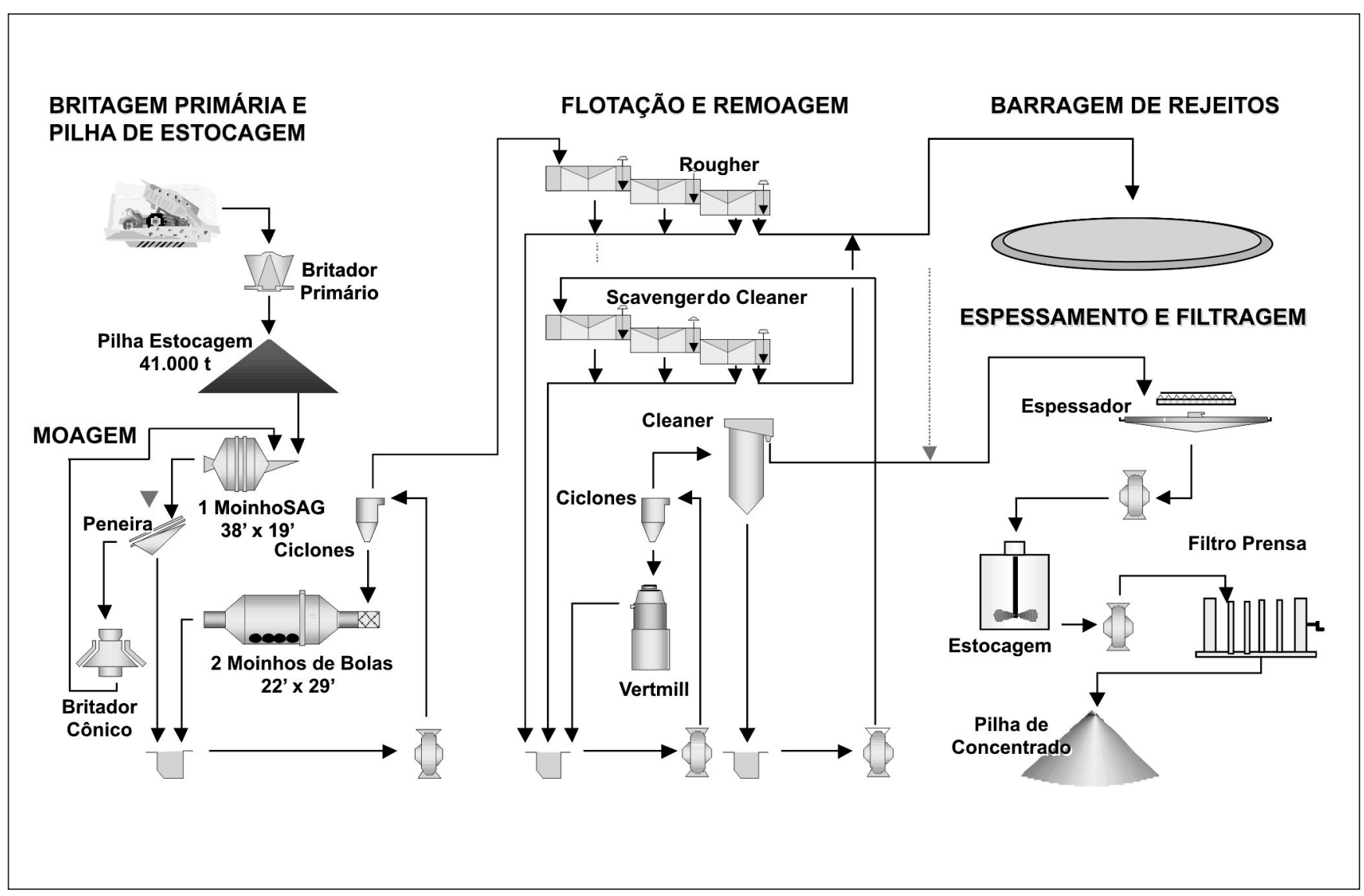

Figura 1 - Fluxograma da usina do Sossego. 
melhor equilíbrio entre os consumos de energia nas etapas de moagem SAG, rebritagem e moagem de bolas.

Após a implementação de tais medidas, foi iniciado um estudo de variabilidade, cujo objetivo foi determinar a influência da dureza do minério na vazão de alimentação do circuito de moagem. A seguir, são apresentados os resultados de tais estudos, bem como os resultados das simulações computacionais visando a otimizar as variáveis operacionais e de processo do circuito.

\section{Materiais e métodos Caracterização do minério}

Amostras de minério de várias campanhas de sondagem, com diâmetros de 1", 2" e 6", assim como amostras de alimentação do moinho SAG, foram ensaiadas visando à determinação de sua dureza para a moagem $\mathrm{SAG}$, utilizando o ensaio desenvolvido pelo JKMRCJulius Kruttschnitt Mineral Research Centre (Napier-Munn, 1999), denominado Drop Weight Test (DWT). As amostras de 6" e 3 amostras de alimentação do SAG foram ensaiadas segundo o método DWT completo (DWT-C), que inclui as seguintes frações granulométricas:

- $63,0 \times 53,0 \mathrm{~mm}$

- $45,0 \times 37,5 \mathrm{~mm}$

- $31,5 \times 26,5 \mathrm{~mm}$

- $22,4 \times 19,0 \mathrm{~mm}$

- $16,0 \times 13,2 \mathrm{~mm}$

Para as amostras de 1", 2" e demais amostras de alimentação do SAG, foi utilizado o teste denominado de DWT simplificado (DWT-S), seguindo a metodologia desenvolvida por Chieregati e Delboni (Chieregati, 2001), mediante ensaio dos fragmentos contidos na fração $22,4 \times 19,0 \mathrm{~mm}$.

A campanha de caracterização incluiu 282 testes, conforme descrito a seguir:

- 52 amostras de furos de sonda de 6".
- 113 amostras de furos de sonda de 2".

- 78 amostras de furos de sonda de 1".

- 39 amostras de alimentação do moinho SAG.

As amostras de 2" e 6" foram coletadas nas caixas de testemunhos em pontos localizados, retirando-se $30 \mathrm{~cm}$ de fragmentos contínuos, ao longo de furos que representavam toda a vida útil da cava. As amostras de 1" foram compostas com fragmentos de $5 \mathrm{~cm}$ em intervalos de $10 \mathrm{~m}$ nas caixas de testemunhos, representando as cavas dos anos de 2007 e 2008. As amostras da alimentação do SAG foram obtidas na correia transportadora que alimenta o moinho.

\section{Modelagem do circuito de moagem}

Os dados experimentais que serviram de base para a modelagem do circuito de moagem foram obtidos em duas campanhas de amostragem (2006 e 2007), conduzidas seguindo os protocolos descritos em Napier-Munn et al. (1999). Antes da amostragem, as condições operacionais foram monitoradas para assegurar que o circuito estivesse sob regime estável de operação.

Durante um período de duas horas, nove fluxos da planta foram amostrados de forma incremental, seguindo resultados de cálculos das massas mínimas de amostra a serem coletadas em cada ponto. Os seguintes pontos foram amostrados:

- Alimentação do moinho SAG.

- Fração retida na peneira de descarga do moinho SAG (alimentação dos rebritadores).

- Produto dos rebritadores.

- Alimentação dos ciclones.

- Overflow dos ciclones e alimentação da flotação.

- Underflow dos ciclones e alimentação dos moinhos de bolas.

- Produto dos moinhos de bolas.
Devido a limitações, não foi possível amostrar o fluxo passante da peneira de descarga do SAG. Os valores de vazão de sólidos e água desse fluxo foram posteriormente estimados por balanço de massas.

Imediatamente após a amostragem, o moinho SAG foi parado na forma de crash stop (parada simultânea da correia de alimentação, água de alimentação e do moinho), para medição da carga e dimensões internas do moinho. Em seguida rodou-se o moinho em vazio para medição da carga de bolas. As dimensões dos moinhos de bolas e ciclones foram determinadas em paradas da planta dois dias antes das amostragens.

As amostras foram analisadas no laboratório de processo localizado na própria usina do Sossego. Foram determinadas porcentagens de sólidos e distribuições granulométricas de todos os fluxos. Uma amostra da alimentação do SAG foi submetida à determinação do Work Index de Bond, índice de abrasão de Bond e DWT-C.

Foram registrados, também, durante as amostragens, os principais dados operacionais do sistema supervisório da usina.

Os dados das amostragens foram balanceados utilizando-se o algoritmo de balanço de massas do software JKSimMet versão 5.1 (JKTech Pty Ltd., Queensland, Austrália). Após ajuste do balanço de massas, os modelos de moagem, britagem e classificação do JKSimMet foram calibrados para o circuito.

Após a calibração dos modelos, foi realizada uma série de simulações para a identificação de cenário para a otimização do circuito. As principais alternativas estudadas foram as seguintes: inclusão de britagem secundária da fração $-5+3$ "da britagem primária, abertura da tela da peneira de descarga do SAG, mudança na geometria dos ciclones, além de análises de sensibilidade sobre dureza e granulometria do minério, carga e tamanho de bolas no SAG. 


\section{Resultados e discussão}

\section{Caracterização do minério}

Os resultados dos testes de DWT-S resultam em valores menores de $\mathrm{A}$ *b (minério mais duro) do que os correspondentes obtidos com o DWT-C. Essa diferença se deve ao fato de que, via de regra, a resistência do fragmento aumenta com a diminuição de tamanho, devido à menor presença de falhas, planos naturais de fraqueza e fraturas induzidas pelo desmonte. Desta forma é necessário calcular o equivalente $A * b$ para o DWT-C com base no resultado do DWTS. Tal procedimento foi conduzido nas amostras ensaiadas de 1", 2" e 6".

A Figura 2 ilustra os resultados obtidos com os respectivos ajustes. Fica claro que as amostras de 2 e 6 " são mais heterogêneas que as de 1" e alimentação do SAG. As análises das descrições dos furos de sonda indicam que algumas dessas amostras mais friáveis eram compostas basicamente de calcopirita pura. As amostras de 1" e alimentação do SAG mostraram uma distribuição mais homogênea. Esse fato é atribuído à forma como foram compostas tais amostras, representando de forma mais fiel a alimentação real do SAG, a qual é composta por fragmentos de calcopirita diluídos nas rochas encaixantes, basicamente granitos. É interessante observar, também, que as amostras de alimentação do SAG são relativamente mais competentes que os outros grupos. Tal aspecto sugere que a variabilidade do minério não é o principal fator que impacta as variações de taxa observadas no moinho SAG.

\section{Modelagem do circuito de moagem}

Os dados utilizados no balanço de massas indicaram boa qualidade dos dados, fato esse confirmado pelos erros obtidos para as vazões mássicas, de água e de granulometria ao redor de cada equipamento. Os dados balanceados geraram um bom ajuste para os modelos selecionados, indicando a boa qualidade do trabalho experimental.
Cabe destacar que em uma das amostragens, o moinho estava rodando com $67 \%$ de sua velocidade crítica, enquanto ele usualmente roda com $80 \%$ da velocidade crítica. Isso se deve ao fato de o $\mathrm{P}_{80}$, em uma das amostragens, estar em 3,5", contra valores médios de 5". Em função disso, os modelos ajustados cobrem uma ampla faixa de granulometrias de alimentação e velocidades do moinho SAG, o que resultou em um modelo robusto para o moinho SAG.

Outro ponto interessante observado na amostragem foi a quantidade de material menor que $0,210 \mathrm{~mm}$ passante na peneira de descarga do SAG, que ficou em torno de $60 \%$ da massa total nesse ponto, portanto pronta para alimentar a flotação. Isso ilustra a grande quantidade de finos gerada pelo moinho SAG.

Com base no modelo calibrado, foram realizadas simulações visando avaliar o impacto de variáveis operacionais de forma individual sobre a taxa do circuito. Os resultados mostraram que:

- A britagem da fração $-5 "+3$ " do britador primário acarreta em aumento de 13 a $29 \%$ na capacidade do circuito.

- A mudança na abertura de 13 para 19 $\mathrm{mm}$ da tela na peneira de descarga do SAG resulta em queda na carga circulante dos rebritadores de 19 a $25 \%$.
- A mudança na geometria dos ciclones indica um produto mais grosso do circuito e, consequentemente, queda significativa na carga circulante do moinho de bolas.

- A variação de resistência do minério indica oscilação de até $6 \%$ na capacidade do circuito para o plano de lavra de 2007 e 2008.

- O afinamento da granulometria de alimentação $\left(\mathrm{P}_{80}\right.$ de 5,5" para 4,5") resulta em aumento de $10 \%$ na capacidade do circuito.

- O aumento de 133 para 140 mm no diâmetro de bolas do moinho SAG resulta em aumento de $8 \%$ na capacidade do circuito.

- O aumento de 133 para 140 mm no diâmetro de bolas do moinho SAG resulta em aumento de $10 \%$ no $\mathrm{P}_{80}$ de alimentação da flotação.

Algumas das mudanças apresentadas anteriormente, como mudança da geometria dos ciclones e granulometria de alimentação do SAG, foram implementadas e os resultados obtidos foram consistentes com os indicados pelas respectivas simulações. A variação observada na vazão em função das características de resistência do minério também apresentara consistência com as previsões

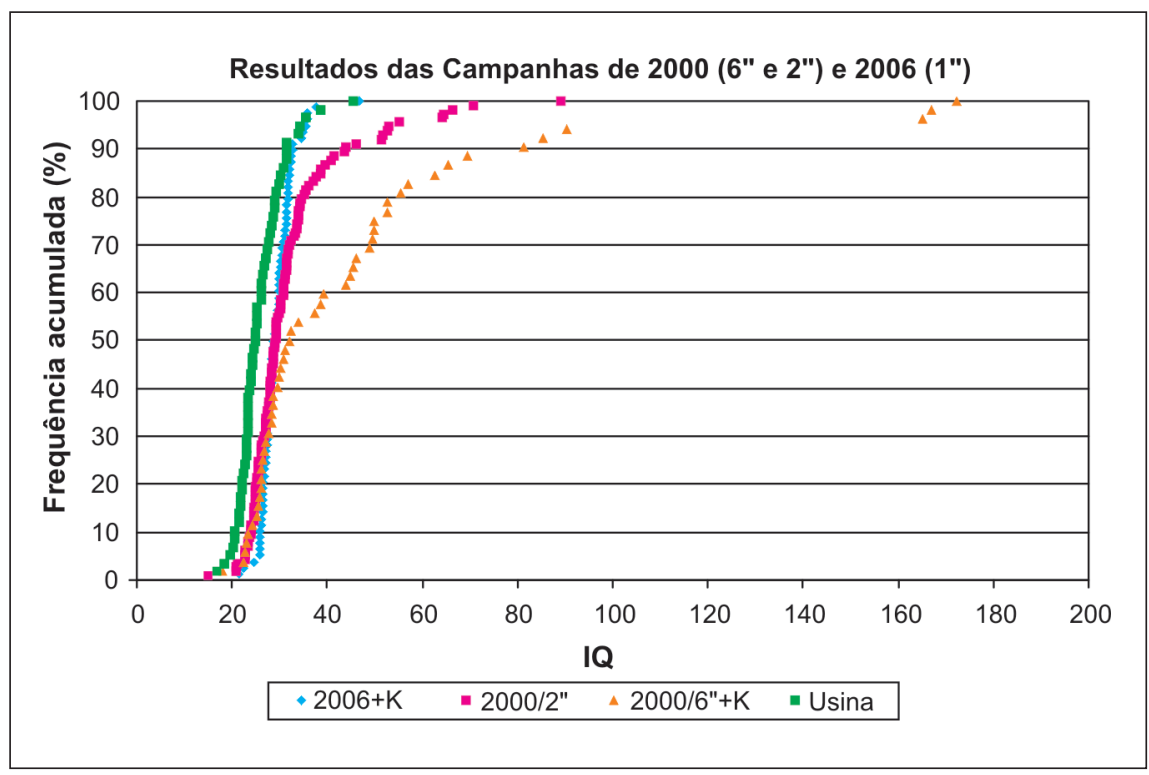

Figura 2 - Distribuições dos resultados de DWT. 
das simulações. O tamanho de bolas do moinho SAG também foi mudado. No entanto, não foi possível avaliar o impacto em planta, pois essa adequação foi realizada no mesmo período em que se alterou a abertura das grelhas, impossibilitando a avaliação em separado das duas variáveis.

As simulações também indicaram que as bolas recicladas de 4" poderiam ser usadas no moinho de bolas, acarretando apenas um pequeno engrossamento, da ordem de $10 \%$, na alimentação da flotação. Essa ação, em conjunto com a adequação da geometria dos ciclones, ajudou a reduzir a carga circulante dos moinhos de bolas, além de aumentar a recuperação metalúrgica da flotação. Isso se deve ao fato de que as perdas de cobre na flotação estão relacionadas a partículas abaixo de $8 \mu \mathrm{m}$, sendo que $30 \%$ da massa de cobre alimentada na flotação se encontrava abaixo dessa malha, enquanto apenas $4 \%$ encontrava-se acima de $210 \mu \mathrm{m}$ (malha acima da qual se observam problemas de liberação). A distribuição mais adequada da granulometria de alimentação da flotação levou a ganhos de $1 \%$ na recuperação global da planta.

\section{Conclusões}

A campanha de ensaios realizada para determinação das características de resistência à moagem de amostras resultou em uma base de dados abrangente sobre os tipos de minérios da jazida do Sossego. Essa base de dados incluiu amostras provenientes, tanto de testemunhos de sondagens, como de alíquo- tas de alimentação do moinho SAG. Os resultados indicaram uma variação relativamente pequena entre as características das amostras.

Simulações de desempenho do circuito de moagem mostraram que tais variações de características resultaram em alterações de até $6 \%$ na vazão de alimentação. Embora esse valor possa ser considerado pequeno, os resultados obtidos permitem a melhor preparação de planos de lavra, bem como torna mais realistas as previsões de produção.

As simulações realizadas a partir do modelo construído com base em amostragens na usina do Sossego mostraram a influência individual ou combinada de uma série de variáveis de operação do circuito. Tais influências foram avaliadas, não apenas em termos de vazão de alimentação do circuito, mas, também, nas características do produto do circuito e sua influência no desempenho da etapa de flotação.

Alternativas para expansão de capacidade puderam também ser hierarquizadas através de simulações.

\section{Agradecimentos}

Os autores agradecem a empresa Vale pelo apoio na realização e publicação desse trabalho.

\section{Referências bibliográficas}

CHIEREGATI, A.C. Novo método de caracterização tecnológica para cominuição de minérios. São Paulo: Universidade de São Paulo, 2001. (Tese de Mestrado).

DELBONI, H. Diagnóstico de desempenho da usina de moagem do Sossego. HDA Serviços Ltda, Outubro de 2004. (Relatório interno).

DELBONI, H., ROSA, M., BERGERMAN, M., NARDI, R. Optimization of the Sossego SAG Mill. In: Advances in autogenous and semi autogenous grinding technology, 2006, Vancouver. p. I39-I50. 1 CD-ROM.

NANKRAN, M., BERGERMAN, M., FONSECA, R., QUEIROZ, A., GERALDO, J. Abertura do circuito de moagem da usina do Sossego. In: ENCONTRO NACIONAL DE TRATAMENTO DE MINÉRIOS, 22. Ouro Preto, Brasil, Novembro de 2007.

NANKRAN, M., BERGERMAN, M., MIRANDA, A., OLIVEIRA, J., SOUZA, M., BATISTA FILHO, J., CARDOSO, W. Controle operacional da usina do Sossego, In: ENCONTRO NACIONAL DE TRATAMENTO DE MINÉRIOS, 22. Ouro Preto, Brasil, Novembro de 2007.

NAPIER-MUNN, T.J. et al. Mineral comminution circuits: their operation and optimization. Indooroopilly: Julius Kruttschnitt Mineral Research Centre/University of Queensland, 1999 (JKMRC Monograph Series in Mining and Mineral Processing).

Artigo recebido em 20/03/2008 e aprovado em 15/06/2008.

\section{A REM tem novo endereço: Rua Carlos Walter Marinho Campos, 57 Bairro: Vila Itacolomy 35400-000 - Ouro Preto - MG}

